\title{
TREE COMPONENT DEMOGRAPHY IN AN UPPER MONTANE MIXED OMBROPHILOUS FOREST UNDER CHRONIC ANTHROPOGENIC DISTURBANCES ${ }^{1}$
}

\author{
Juliana Pizutti Dallabrida ${ }^{2 *}$, Aline Pereira Cruz ${ }^{2}$, Chayane Cristina de Souza ${ }^{2}$, Mariele Alves Ferrer da \\ Silva $^{2}$, Vanessa de Fátima Soboleski², Rodineli Loebens ${ }^{2}$, Fernando Buzzi Júnior ${ }^{3}$, Ana Carolina da Silva ${ }^{4}$ \\ and Pedro Higuchi ${ }^{4}$

\footnotetext{
${ }^{1}$ Received on 23.11.2015 accepted for publication on 31.05.2017.

${ }^{2}$ Universidade do Estado de Santa Catarina, Programa de Pós-Graduação em Engenharia Florestal, Lages, Santa Catarina, Brasil.E-mail: <juli_ajuri@hotmail.com>, <a.line_cruz@yahoo.com.br>, $<$ chayanesouza8@gmail.com>, $<$ mariele.ferrer@hotmail.com>, $<$ vanessasoboleski@hotmail.com>and<rhodineli@hotmail.com>.

${ }^{3}$ Universidade do Estado de Santa Catarina,Graduação em Engenharia Florestal,Lages, Santa Catarina, Brasil. E-mail: $<$ fernandobuzzijunior@gmail.com>.

${ }^{4}$ Universidade do Estado de Santa Catarina,Departamento em Engenharia Florestal, Lages, Santa Catarina, Brasil. E-mail: $<$ carol_sil4@yahoo.com.br>and<higuchip@gmail.com>.

*Corresponding author.
}

\begin{abstract}
The objective of this study was to understand the dynamics of an Upper Montane Mixed Ombrophilous Forest in the municipality of Painel, Santa Catarina, Brazil, where low-level but chronic disturbances, such as extensive cattle grazing and exploitation of non-timber products, are common practices. In 2008, permanent plots were allocated and all tree species with circumference at breast height $(\mathrm{CBH})$ equal to or greater than $15.7 \mathrm{~cm}$ were measured. A second survey was conducted in 2014 by the same methodology. Mortality, recruitment, and basal area gain and loss rates were then determined. The tree species were classified into regeneration guilds and proportion tests were conducted to verify the associations between dynamics patterns and guilds. The community has shown a $2.3 \%$.year ${ }^{-1}$ average mortality rate, a $2.2 \%$.year ${ }^{-1}$ average recruitment rate, a $3.0 \%$.year ${ }^{-1}$ average basal area gain rate and a $1.8 \%$.year $^{-1}$ average basal area loss rate. Species number has decreased from 55 to 48 . Regeneration guilds have shown different dynamics patterns, where the pioneers have shown the greatest mortality $(19.9 \%)$ and recruitment $(15.6 \%)$ proportion and reduced representativeness in shade-tolerant climax species. Therefore, floristic impoverishment and higher representativeness reduction of late species have been observed. We have concluded that despite disturbances do not result in structural deterioration, they have the potential to slow the succession process and cause a floristic homogenization in the area under study.
\end{abstract}

Keywords: Mixed Ombrophilous Forest; Temporal changes; Forest succession.

\section{DEMOGRAFIA DO COMPONENTE ARBÓREO EM UMA FLORESTA OMBRófILA MISTA ALTO-MONTANA SUJEITA A PERTURBAÇÕES ANTRÓPICAS CRÔNICAS}

RESUMO-O objetivo do presente trabalho foi compreender a dinâmica de um fragmento de Floresta Ombrófila Mista Alto-Montana, no municipio de Painel, em Santa Catarina, onde perturbações de baixa intensidade, porém crônicas, tais como pastoreio extensivo e exploração de produtos não-madeireiros, são práticas comuns. Em 2008, foram instaladas parcelas permanentes, onde foram mensurados todos os indivíduos arbóreos com circunferência a altura do peito (CAP) igual ou superior a 15,7 cm. Em 2014, foi realizado o segundo inventário da comunidade arbórea, adotando-se a mesma metodologia. Foram calculadas as taxas de mortalidade, recrutamento e ganho e perda em área basal. Os indivíduos inventariados foram classificados em guildas de regeneração e foram realizados testes de proporção a fim de verificar a associação entre os padrões de 
dinâmica e as guildas. Para a comunidade, as taxas médias de mortalidade e recrutamento foram de 2,3\%.ano-1 $e$ 2,2\%.ano ${ }^{-1}$, respectivamente, e as taxas médias de ganho e perda em área basal foram de 3,0\%.ano $e$ 1,8\%.ano ${ }^{-1}$, respectivamente. Ocorreu a redução de espécies, passando de 55 para 48. As guildas de regeneração apresentaram diferentes padrões de dinâmica, com as pioneiras com maior proporção de mortalidade (19,9\%) e recrutamento $(15,6 \%)$ e com redução da representatividade do grupo de clímax tolerante à sombra. Desta forma, observou-se um empobrecimento florístico e maior redução na representatividade de espécies tardias. Conclui-se que na área de estudo, apesar das perturbações não resultarem em uma degradação estrutural, essas têm o potencial de retardar o processo de sucessão e causar uma homogeneização florística.

Palavras-Chave: Floresta com Araucária; Mudança temporal; Sucessão florestal.

\section{INTRODUCTION}

The southern Brazilian Mixed Ombrophilous Forest (MOF) has a history of intense anthropic disturbances caused by agricultural occupation, plant extractivism and the establishment of agricultural enterprises (Kozera et al., 2006), which has reduced its original coverage area (Ribeiro et al., 2009). However, the forests reduction process has been decelerated since the end of the last century so that this scenario has changed in some regions with the increase of the secondary forests area (Baptista and Rudel, 2006). This change has occurred in response to the creation of federal environmental laws and the social and economic forces that resulted in rural exodus (Baptista and Rudel, 2006). Yet it is observed that the disturbance regime in MOF areas currently shows different characteristics, where the more intense and punctual impacts that used to be frequent have been supplanted by low-level but chronic disturbances, such as cattle presence (Sampaio and Guarino, 2007; Silva et al., 2012) and the exploitation of non-timber resources (Mello and Peroni, 2015). In fact, impacts caused by traditional use practices have an old history in MOF areas so that Reis et al. (2014) consider all its regional landscape to be shaped that way since the pre-Columbian societies. This whole history represents a frequent forest pattern in different parts of the world (Lewis et al., 2015) as one of the Anthropocene facets, a period in which anthropic influence has become universal (Corlet, 2014). Understanding how these ecosystems work in such context is therefore necessary for defining conservation and sustainable use strategies (Lugo, 2015) related to the current reality of forest remnants.

Among ecological studies that lead to relevant conclusions on the functioning and successional trajectory of forests, we highlight the ones evaluating tree species demography. Hence, approaches of this nature have been carried out in a large number of phytophysiognomies in Brazil (Formento et al., 2004; Oliveira Filho et al., 2007; Schorn and Galvão, 2009; Figueiredo Filho et al., 2010; Mews et al., 2011). These studies have demonstrated different patterns that, in general, reflect the existing history of disturbances. In forests with no recent acute disturbances, such as those related to extreme climatic events or anthropogenic actions resulting in the formation of large gaps or deforestation, there is a tendency to increase the representativeness of shade-tolerant late or climax species and to maintain or increase both richness and basal area gain (Formento et al., 2004; Oliveira Filho et al., 2007; Schorn and Galvão, 2009; Sanquetta et al., 2010; Salami et al., 2014). On the other hand, it is possible to observe a degradation process, synthesized by the reduction of individuals and basal area, in forests subject to intense or chronic disturbances. Some authors have observed these patterns in forest remnants subject to cattle presence and selective logging in Minas Gerais, Brazil (Machado and Oliveira Filho, 2010); in a transition forest between the Brazilian Cerrado and the Amazon, suggesting the influence of a strong drought as a determining factor (Mews et al., 2011); and in alluvial forest remnants subject to anthropic disturbances, after a heavy flood in the south of Minas Gerais, Brazil (Silva et al., 2011). Moreover, studies carried out in transformed landscapes have shown that disturbances caused by selective logging without planning and/or cattle grazing have negatively influenced richness by favoring pioneer species rather than late ones and therefore either slowing the process of succession down or limiting it (Clark and Covey, 2012; Zamorano-Elgueta et al., 2014; Arroyo Rodríguez et al., 2015). However, the ecological value of cultural landscapes traditionally managed by rural populations should not be ignored due to the fact that many tree species are able to remain in such places (Mello and Peroni, 2015) and that these areas can perform more ecosystem functions than those exclusively used for agrosilvopastoral purposes.

Revista Árvore. 2017;41(3):e410312 
Considering that researches on the impact of chronic anthropogenic disturbances may contribute to the definition of conservation strategies for forest remnants, this study aimed to analyze the tree component demographic changes in an Upper Montane Mixed Ombrophilous Forest area subject to such conditions. The goal was to identify its demographic pattern by testing the hypothesis that chronic anthropogenic disturbances cause a process of structural and floristic degradation. We have also verified which species are favored or not by such scenario.

\section{MATERIALAND METHODS}

The study was carried out in the municipality of Painel, Southern Plateau of the State of Santa Catarina, Brazil, in a fragment located at $27^{\circ} 55^{\prime} 44^{\prime \prime} \mathrm{S}$ and $50^{\circ} 06^{\prime} 18^{\prime \prime} \mathrm{W}$ at 1,399 $\mathrm{m}$ average altitude. According to IBGE (2012), its predominant vegetation is classified as Upper Montane Mixed Ombrophilous Forest. It has a Cfb climate according to Köppen classification and its average annual rainfall varies from 1,200 to $1,900 \mathrm{~mm}$ well distributed along the year (Higuchi et al., 2013). According to the quoted authors, the average annual temperature varies from $15^{\circ} \mathrm{C}$ to $16^{\circ} \mathrm{C}$ and there may be frost and snow during the winter; soils are mainly of basaltic origin, such as lithic neosols and cambisols.

The forest fragment under study is located in a rural property of Farofa, Painel, SC, Brazil, with a total area of near 48.91 ha. According to the owner, this area underwent historical changes due to the anthropic action in the 1970s. At that time, it faced the last extraction period of Araucaria angustifolia (Bertol.) Kuntze, which has left only the individuals with CBH (circumference at breast height) lower than 25 $\mathrm{cm}$ in consortium with native grassland. As one can easily identify this area under a continuous forest cover in the LANDSAT satellite image from 1976, it is assumed that the most intense disturbances have occurred in the late 1970s. Forest succession processes have been occurring since then. This fragment is currently used for extensive cattle grazing, Araucaria nuts collection and Yerba Mate (Ilex paraguariensis A.St.-Hil.) extraction. Cattle have access to this entire forest fragment, but there is a higher concentration of animals in particular areas.

Sampling area was delimited by the floristicstructural study carried out by Higuchi et al. (2013) in 2008 . At that time, $5010 \times 20 \mathrm{~m}\left(200 \mathrm{~m}^{2}\right)$ permanent plots were systematically placed $30 \mathrm{~m}$ distant to each other in random transects aiming to capture all local topographic variation, making 1 ha sampled. All tree species individuals with circumference at breast height $(\mathrm{CBH})$ equal to or higher than $15.7 \mathrm{~cm}$ were measured. Multiple stems tree individuals were measured when the CBHs' root sum squares reached $\mathrm{CBH} \geq 15.7 \mathrm{~cm}$. Such criterion for sampling multiple stems tree individuals was applied for better estimating the basal area. Sampled species were incorporated into the Herbarium of the Santa Catarina State University (LUSC). In 2014, the second inventory was carried out by the same methodology applied to the first one six years before. One of the plots allocated in 2008 has not been found so the study was carried out with 49 plots for both years, making 0.98 ha sampled. Surviving individuals were again measured, dead individuals were registered, and new individuals (recruited) reaching $\mathrm{CBH} \geq 15.7 \mathrm{~cm}$ were identified, measured and tagged.

Data analysis was held by calculating mortality and recruitment rates as well as basal area gain and loss rates based on the number of individuals and on the basal area of the 49 analyzed plots. Therefore, some results obtained in 2008 differ from those obtained by Higuchi et al. (2013), since we have worked with one less plot. As sampling area got reduced by only $2 \%$, there were no significant negative changes in species richness; on the contrary, two species that could not be identified in the first inventory due to leafless have been identified in the current year. Besides that, there were only few changes regarding the individuals' density and basal area.

Mortality and recruitment rates were calculated by the following equations: $\mathrm{M}_{\mathrm{R}}=\left(1-\left(\left(\mathrm{N}_{1}-\mathrm{N}_{\mathrm{d}}\right) / \mathrm{N}_{1}\right)^{1 / \mathrm{t}}\right) \times 100$ (Sheil et al., 1995) and $\mathrm{R}_{\mathrm{R}}=\left(1-\left(1-\mathrm{N}_{\mathrm{r}} / \mathrm{N}_{2}\right)^{1 / t}\right) \times 100$ (Sheil et al, 2000), where $M_{R}=$ mortality rate $\left(\%\right.$.year $\left.{ }^{-1}\right) ; N_{1}=$ initial number of individuals; $\mathrm{t}=$ time; $\mathrm{N}_{\mathrm{d}}=$ number of dead individuals; $\mathrm{R}_{\mathrm{R}}=$ recruitment rate $\left(\%\right.$.year $\left.{ }^{-1}\right) ; \mathrm{N}_{\mathrm{r}}=$ number of recruited individuals; $\mathrm{N}_{2}=$ final number of individuals. Basal area gain and loss rates were determined by the following formulas applied by Oliveira Filho et al. (2007) based on the work of Sheil et al. (1995) and Sheil et al. (2000): $\mathrm{G}_{\mathrm{R}}=\left(1-\left(1-\left(\mathrm{BA}_{\mathrm{G}} / \mathrm{BA}_{2}\right)^{1 / \mathrm{t}}\right) \times 100\right.$; $\mathrm{L}_{\mathrm{R}}=\left(1-\left(\left(\mathrm{BA}_{1}-\mathrm{BA}_{\mathrm{L}}\right) / \mathrm{BA}_{1}\right)^{1 / \mathrm{t}}\right) \times 100$; where, $\mathrm{G}_{\mathrm{R}}=$ basal area gain rate $\left(\%\right.$.year $\left.{ }^{-1}\right) ; \mathrm{BA}_{\mathrm{G}}=$ basal area gain $\left(\mathrm{m}^{2}\right), \mathrm{BA}_{2}=$ final basal area $\left(\mathrm{m}^{2}\right) ; \mathrm{L}_{\mathrm{R}}=$ basal area loss rate $\left(\%\right.$.year $\left.{ }^{-1}\right)$; $\mathrm{BA}_{1}=$ initial basal area $\left(\mathrm{m}^{2}\right) ; \mathrm{BA}_{\mathrm{L}}=$ basal area loss $\left(\mathrm{m}^{2}\right)$ and $\mathrm{t}=$ time. Individuals $\left(\mathrm{Ch}_{\mathrm{N}}\right)$ and basal area $\left(\mathrm{Ch}_{\mathrm{BA}}\right)$ net change rates $\left(\%\right.$.year $\left.{ }^{-1}\right)$ were calculated according 
to the following equations from Korning and Balslev (1994): $\mathrm{Ch}_{\mathrm{N}}=\left(\left(\mathrm{N}_{2} / \mathrm{N}_{1}\right)^{1 / \mathrm{t}}-1\right) \times 100 ; \mathrm{Ch}_{\mathrm{BA}}=\left(\left(\left(\mathrm{BA}_{2} / \mathrm{BA}_{1}\right)^{1 / \mathrm{t}}\right)-\right.$ $1) \times 100$. We have used these equations because they are widely applied in studies on vegetation dynamics, which makes comparative analysis easier.

Individuals were classified according to field notes and literature (Silva et al., 2013; Rech et al., 2015) as pioneer (P), light-demanding climax (LC) and shadetolerant climax (SC) (Swaine and Whitmore, 1988, adapted by Oliveira Filho et al., 1994) regarding tree species regeneration guilds (successional groups). The Test of Equal or Given Proportions was performed with the $\mathrm{R}$ prop.test function based on the chi-square test in order to verify the existence of a significant association between population dynamics patterns (recruitment, survival and mortality) and regeneration guilds. Test's null hypothesis is that the proportion of recruited, survivor and dead individuals from different guilds will be the same. Analyzes were carried out by the R statistical language programming (R Core Team, 2015).

\section{RESULTS}

The tree species community dynamics analysis has shown (Table 1) a $2.3 \%$. year $^{-1}$ mortality rate and a $2.2 \%$.year ${ }^{-1}$ recruitment rate, which has resulted in an average density of 1.371,4 ind.ha ${ }^{-1}$ and 1.343,9 ind.ha ${ }^{-1}$ in 2008 and 2014, respectively, showing only $-0.1 \%$.year $^{-1}$ average net change in the number of individuals. However, basal area average gain rate $\left(3.0 \%\right.$.year $\left.{ }^{-1}\right)$ was superior to the average loss rate $\left(1.8 \%\right.$.year $\left.{ }^{-1}\right)$ therefore resulting in an average basal area of $34.10 \mathrm{~m}^{2} \cdot \mathrm{ha}^{-1}$ and $36.20 \mathrm{~m}^{2} \cdot \mathrm{ha}^{-1}$ in 2008 and 2014 , respectively, showing a $1.2 \%$.year $^{-1}$ average net change.

Regarding species richness, from 2008 to 2014's inventory we have lost nine species (Baccharis caprariifolia DC., Baccharis uncinella DC., Piptocarpha angustifolia Dusén, Solanum sanctaecatharinae Dunal, Solanum sp. 1, Solanum sp. 2, Symphyopappus itatiayensis (Hieron.) R. M. King \& H. Rob., Urera baccifera (L.) Gaudich. ex Wedd. e Vernonanthura discolor (Spreng.) H. Rob.) and gained two (Solanum compressum L.B. Sm. \& Downs e Styrax leprosus Hook. \& Arn.). Species number has thus decreased from 55 in 2008 to 48 in 2014, not considering individuals belonging to the NI group, which have not been identified due to leafless by the time of the inventory.

\footnotetext{
Revista Árvore. 2017;41(3):e410312
}

The highest positive individuals net change values among the most abundant species have been found in the species Myrceugenia glaucescens (Cambess.) D.Legrand \& Kausel $\left(22.2 \%\right.$.year $\left.^{-1}\right)$ with high recruitment rate $\left(18.2 \%\right.$.year $\left.^{-1}\right)$ and no mortality; Drimys angustifolia Miers (2.3\%.year $\left.{ }^{-1}\right)$; and Xylosma ciliatifolia (Clos) Eichler (1.8\%.year-1 ${ }^{-1}$. And the highest positive basal area net change values among the most abundant species have been found in the species Myrceugenia glaucescens $\left(28.8 \%\right.$.year $\left.^{-1}\right)$ with high value due to a $22.4 \%$.year ${ }^{-1}$ basal area gain in recruited individuals and in the surviving individuals growth, and no basal area loss; Xylosma ciliatifolia (3.2\%.year $\left.{ }^{-1}\right)$; Drimys angustifolia $\left(3.1 \%\right.$ year $\left.^{-1}\right)$; and Araucaria angustifolia $\left(2.4 \%\right.$.year $\left.{ }^{-1}\right)$. The highest negative individuals net change values among the most abundant species have been found in the species Berberis laurina Billb. (-4.3\%.year $\left.{ }^{-1}\right) ;$ Myrceugenia regnelliana(O.Berg) D.Legrand\& Kausel (-3.7\%.year$\left.{ }^{1}\right)$; and Dicksonia sellowiana Hook. $\left(-3,2 \%\right.$. ano $\left.^{-1}\right)$. Not only Myrceugenia regnelliana(O.Berg) D.Legrand\& Kausel (-4.1\%.year $\left.{ }^{-1}\right)$ but also Calyptranthes concinna DC. $\left(-2.5 \%\right.$.year $\left.{ }^{-1}\right)$ has shown a high negative basal area net change among the most abundant species.

By analyzing dynamics rates in relation to regeneration guilds one can verify the highest dead proportion $\left(p=4.85 \mathrm{e}^{-07}\right)$ in $\mathrm{P}$ species $(19.9 \%)$, followed by SC (17.0\%) and LC (8.6\%) species (Table 2). P species have also been the most recruited ones $(15.6 \%)$, followed by LC $(8.7 \%)$ and SC species $(8.3 \%)(p=0.0057)$. As a result of such differential dynamics among the analyzed guilds, the SC species have shown the highest representativeness reduction of the forest (the highest difference between mortality and recruitment rates).

\section{DISCUSSION}

The tested hypothesis which chronic disturbances would cause a process of structural degradation characterized by the reduction in abundance and basal area along with the reduction in richness has not been fully accepted. Although 15\% taxonomic impoverishment was noticed as expected, the structural dynamics pattern was practically stable, maintaining both the abundance and the basal area. It is interesting to note that in mature and conserved forests, which is not the case here, a self-thinning process, in which the reduction of both inter and intraspecific competition allows a higher growth of survivor individuals through tree mortality, is expected. Generally, there is also the maintenance of richness 
Tree component demography in an upper...

Table 1 - Species sampled in 2014 from an Upper Montane Mixed Ombrophilous Forest fragment under chronic anthropogenic disturbances and their demographic rates from 2008 and $2014\left[\mathrm{~N}_{2014}=\right.$ number of individuals in $2014 ; \mathrm{M}_{\mathrm{R}}=$ mortality rate $\left(\%\right.$.year $\left.{ }^{-1}\right) ; \mathrm{R}_{\mathrm{R}}=$ recruitment rate $\left(\%\right.$.year $\left.{ }^{-1}\right) ; \mathrm{Ch}_{\mathrm{N}}=$ individuals net change $\left(\%\right.$.year $\left.{ }^{-1}\right) ; \mathrm{BA}_{2014}=$ basal area in $2014\left(\mathrm{~m}^{2}\right) ; \mathrm{L}_{\mathrm{R}}=$ Basal area loss rate $\left(\%\right.$.year $\left.{ }^{-1}\right) ; \mathrm{G}_{\mathrm{R}}=$ basal area gain rate $\left(\%\right.$.year $\left.{ }^{-1}\right) ; \mathrm{Ch}_{\mathrm{BA}}=$ basal area net change (\%.year $\left.{ }^{-1}\right) ; \mathrm{R}=$ Herbarium of Santa Catarina State University (LUSC) register].

Tabela1 - Espécies amostradas no ano de 2014 em um fragmento de Floresta Ombrófila Mista Alto-Montana sob perturbações antrópicas crônicas e taxas demográficas para os anos de 2008 e 2014 para as respectivas espécies e comunidade total [N2014 = número de indivíduos em 2014; TM= taxa de mortalidade (\%.ano-1); TR= taxa de recrutamento (\%.ano-1); $\mathrm{ChN}=$ mudança líquida em número de indivíduos (\%.ano-1); AB2014 = área basal em $2014(\mathrm{~m} 2) ;$ $\mathrm{TP}=$ taxa de perda em área basal (\%.ano-1); $\mathrm{TG}=$ taxa de ganho em área basal (\%.ano-1); $\mathrm{ChAB}=$ mudança líquida em área basal (\%.ano-1); $\mathrm{R}$ = registro no Herbário da Universidade do Estado de Santa Catarina, LUSC].

\begin{tabular}{|c|c|c|c|c|c|c|c|c|c|}
\hline Species & $\mathrm{N}_{2014}{ }^{*}$ & $\mathrm{M}_{\mathrm{R}}$ & $R_{R}$ & $\mathrm{Ch}_{\mathrm{N}}$ & $\mathrm{BA}_{2014^{*}}$ & $\mathrm{~L}_{\mathrm{R}}$ & $\mathrm{G}_{\mathrm{R}}$ & $\mathrm{Ch}_{\mathrm{BA}}$ & $\mathrm{R}$ \\
\hline $\begin{array}{l}\text { Myrceugenia euosma } \\
\text { (O.Berg) D.Legrand }\end{array}$ & 313 & 1.2 & 1.2 & 0.0 & 4.70 & 1.7 & 2.2 & 0.6 & D2573 \\
\hline $\begin{array}{l}\text { Acca sellowiana } \\
\text { (O.Berg) Burret }\end{array}$ & 174 & 1.4 & 2.4 & 1.1 & 2.01 & 1.8 & 3.6 & 1.9 & D2570 \\
\hline $\begin{array}{l}\text { Araucaria angustifolia } \\
\text { (Bertol.) Kuntze }\end{array}$ & 167 & 0.3 & 0.8 & 0.5 & 12.08 & 0.1 & 2.4 & 2.4 & D2559 \\
\hline Drimys brasiliensis Miers & 110 & 2.6 & 1.4 & -1.2 & 1.68 & 3.4 & 3.2 & -0.3 & D2593 \\
\hline $\begin{array}{l}\text { Myrceugenia oxysepala } \\
\text { (Burret) D.Legrand \& Kausel }\end{array}$ & 97 & 1.8 & 1.8 & 0.0 & 1.23 & 2.8 & 2.6 & -0.2 & D2576 \\
\hline Drimys angustifolia Miers & 55 & 2.2 & 4.4 & 2.3 & 0.93 & 2.3 & 5.2 & 3.1 & - \\
\hline Dicksonia sellowiana Hook. & 51 & 3.2 & 0.0 & -3.2 & 3.45 & 3.6 & 2.8 & -0.9 & D2564 \\
\hline Ocotea pulchella Mart. & 46 & 1.8 & 1.1 & -0.7 & 1.31 & 1.6 & 3.5 & 1.9 & D2568 \\
\hline Ilex paraguariensis A.St.-Hil. & 41 & 3.4 & 2.6 & -0.8 & 0.33 & 2.9 & 3.1 & 0.2 & D2556 \\
\hline $\begin{array}{l}\text { Cinnamomum amoenum } \\
\text { (Nees) Kosterm }\end{array}$ & 33 & 0.5 & 0.0 & -0.5 & 2.97 & 0.2 & 2.3 & 2.2 & D2609 \\
\hline $\begin{array}{l}\text { Schinus polygamus } \\
\text { (Cav.) Cabrera }\end{array}$ & 28 & 1.3 & 1.9 & 0.6 & 0.31 & 0.7 & 2.2 & 1.5 & D2554 \\
\hline Calyptranthes concinna DC. & 21 & 3.3 & 2.5 & -0.8 & 0.21 & 6.8 & 4.4 & -2.5 & D2572 \\
\hline $\begin{array}{l}\text { Myrceugenia regnelliana } \\
\text { (O.Berg) D.Legrand\& Kausel }\end{array}$ & 20 & 3.7 & 0.0 & -3.7 & 0.17 & 6.3 & 2.3 & -4.1 & $\mathrm{D} 2575$ \\
\hline $\begin{array}{l}\text { Myrceugenia myrcioides } \\
\text { (Cambess.) O.Berg }\end{array}$ & 13 & 3.7 & 1.3 & -2.4 & 0.12 & 3.1 & 2.1 & -1.0 & D2574 \\
\hline Prunus myrtifolia (L.) Urb. & 13 & 2.5 & 1.3 & -1.2 & 0.29 & 1.6 & 2.8 & 1.3 & D2581 \\
\hline Ilex microdonta Reissek & 12 & 1.4 & 1.4 & 0.0 & 0.33 & 2.6 & 2.1 & -0.5 & D2555 \\
\hline Berberis laurina Billb. & 10 & 5.9 & 1.7 & -4.3 & 0.05 & 8.0 & 6.7 & -1.4 & D2561 \\
\hline $\begin{array}{l}\text { Myrceugenia glaucescens } \\
\text { (Cambess.) D.Legrand \& Kausel }\end{array}$ & 10 & 0.0 & 18.2 & 22.2 & 0.04 & 0.0 & 22.4 & 28.8 & - \\
\hline $\begin{array}{l}\text { Xylosma ciliatifolia } \\
\text { (Clos) Eichler }\end{array}$ & 10 & 0.0 & 1.7 & 1.8 & 0.08 & 0.0 & 3.1 & 3.2 & - \\
\hline Zanthoxylum rhoifolium Lam. & 10 & 1.6 & 0.0 & -1.6 & 0.18 & 3.9 & 4.6 & 0.7 & D2583 \\
\hline Inga lentiscifolia Benth. & 9 & 1.7 & 0.0 & -1.7 & 0.18 & 0.7 & 1.9 & 1.2 & D2565 \\
\hline Myrsine coriacea & 8 & 2.2 & 2.2 & 0.0 & 0.42 & 1.0 & 2.5 & 1.5 & D2578 \\
\hline (Sw.) Roem. \& Schult. & & & & & & & & & \\
\hline Persea willdenowii Kosterm. & 7 & 0.0 & 0.0 & 0.0 & 0.48 & 0.0 & 2.2 & 2.3 & D2569 \\
\hline $\begin{array}{l}\text { Blepharocalyx salicifolius } \\
\text { (Kunth) O.Berg }\end{array}$ & 5 & 3.7 & 3.7 & 0.0 & 0.08 & 2.3 & 5.8 & 3.6 & D2571 \\
\hline Ilex taubertiana Loes. & 5 & 0.0 & 0.0 & 0.0 & 0.32 & 0.0 & 0.9 & 0.9 & D2557 \\
\hline Mimosa scabrella Benth. & 5 & 9.3 & 0.0 & -9.3 & 0.96 & 8.5 & 4.0 & -4.7 & D2566 \\
\hline NI & 4 & 16.7 & 20.6 & 4.9 & 0.02 & 30.1 & 10.6 & -21.9 & \\
\hline Solanum variabile Mart. & 4 & 10.9 & 4.7 & -6.5 & 0.01 & 13.0 & 9.5 & -3.9 & - \\
\hline $\begin{array}{l}\text { Dasyphyllum spinescens } \\
\text { (Less.) Cabrera }\end{array}$ & 3 & 6.5 & 6.5 & 0.0 & 0.04 & 7.0 & 11.1 & 4.7 & - \\
\hline Myrrhinium & 3 & 6.5 & 6.5 & 0.0 & 0.02 & 8.5 & 3.0 & -5.6 & D2577 \\
\hline
\end{tabular}


Table 1...

Tabela 1...

\begin{tabular}{|c|c|c|c|c|c|c|c|c|c|}
\hline \multicolumn{10}{|l|}{ atropurpureum Schott } \\
\hline Solanum compressum & 3 & 0.0 & 100.0 & 0.0 & 0.01 & 0.0 & 100.0 & 0.0 & - \\
\hline \multicolumn{10}{|l|}{ L.B. Sm. \& Downs } \\
\hline Solanum ramulosum Sendtn. & 3 & 16.7 & 0.0 & -16.7 & 0.03 & 16.1 & 4.3 & -12.4 & D2589 \\
\hline \multicolumn{10}{|l|}{ (A.St.-Hil.) Radlk. } \\
\hline Citharexylum solanaceum Cham & n. 2 & 0.0 & 10.9 & 12.3 & 0.01 & 0.0 & 14.8 & 17.4 & - \\
\hline Rhamnus sphaerosperma $\mathrm{Sw}$. & 2 & 0.0 & 10.9 & 12.3 & 0.00 & 0.0 & 12.4 & 14.1 & D2580 \\
\hline Solanum mauritianum Scop. & 2 & 0.0 & 10.9 & 12.3 & 0.04 & 2.9 & 3.1 & 0.3 & D2585 \\
\hline $\begin{array}{l}\text { Solanum pabstii } \\
\text { L.B.Sm. \& Downs }\end{array}$ & \multicolumn{9}{|c|}{ L.B.Sm. \& Downs } \\
\hline Solanum paranense Dusén & 2 & 10.9 & 0.0 & -10.9 & 0.01 & 12.7 & 3.1 & -9.9 & D2587 \\
\hline Styrax leprosus Hook. \& Arn. & 2 & 0.0 & 100.0 & 0.0 & 0.00 & 0.0 & 100.0 & 0.0 & - \\
\hline $\begin{array}{l}\text { Baccharis dentata } \\
\text { (Vell.) G.M.Barroso }\end{array}$ & 1 & 100.0 & 100,0 & $-10,9$ & 0,00 & 100,0 & 100,0 & $-13,9$ & - \\
\hline $\begin{array}{l}\text { Zanthoxylum kleinii } \\
\text { (R.S.Cowan) P.G.Waterman }\end{array}$ & 1 & 10.9 & 0,0 & $-10,9$ & 0,18 & 0,3 & 1,5 & 1,2 & D2582 \\
\hline \multicolumn{10}{|l|}{ xanthocarpa (Mart.) O.Berg } \\
\hline Clethra scabra Pers. & 1 & 100.0 & 100.0 & 0.0 & 0.00 & 100.0 & 100.0 & -10.9 & D2563 \\
\hline Ilex theezans Mart. ex Reissek & 1 & 0.0 & 0.0 & 0.0 & 0.00 & 0.0 & 3.7 & 3.9 & D2558 \\
\hline Lauraceae sp. 1 & 1 & 0.0 & 0.0 & 0.0 & 0.03 & 0.0 & 0.3 & 0.3 & - \\
\hline $\begin{array}{l}\text { Maytenus ilicifolia } \\
\text { Mart. ex Reissek }\end{array}$ & 1 & 0.0 & 0.0 & 0.0 & 0.00 & 0.0 & 13.5 & 15.6 & D2562 \\
\hline $\begin{array}{l}\text { Myrcia hartwegiana } \\
\text { (O.Berg) Kiaersk. }\end{array}$ & 1 & 0.0 & 0.0 & 0.0 & 0.01 & 0.0 & 2.8 & 2.9 & - \\
\hline Solanum pseudoquina A.St.-Hil & 1. 1 & 100.0 & 100.0 & 0.0 & 0.00 & 100.0 & 100.0 & -33.1 & D2588 \\
\hline Symplocos sp. & 1 & 0.0 & 0.0 & 0.0 & 0.00 & 0.0 & 6.9 & 7.4 & - \\
\hline Community Total & 1317 & 2.1 & 1.8 & -0.3 & 35.43 & 1.8 & 2.7 & 1.0 & \\
\hline $\mathrm{IC}_{95 \%}$ lower & 181.2 & 1.9 & 1.6 & -0.8 & 31.7 & 1.2 & 2.7 & 0.5 & \\
\hline Average $^{+}$ & 343.9 & 2.3 & 2.2 & -0.1 & 36.2 & 1.8 & 3.0 & 1.2 & \\
\hline $\mathrm{IC}_{95 \%}$ upper & 506.5 & 2.8 & 2.7 & 0.5 & 40.6 & 2.5 & 3.3 & 1.8 & \\
\hline
\end{tabular}

${ }^{*}$ Average values of $N_{2014}$ and $B A_{2014}$ for the community are expressed in ha (ind.ha ${ }^{1}$ and $\mathrm{m}^{2}$.ha ${ }^{-1}$ ). ${ }^{+}$Community average values considering all 49 analyzed plot.

Table 2 - Number of individuals, dead and recruited individuals percentage (values between parentheses) and individuals net change $\left(\mathrm{Ch}_{\sqrt{ }}\right)$ per regeneration guild in an Upper Montane Mixed Ombrophilous Forest fragment under chronic anthropogenic disturbances, where $N_{1}=$ number of trees in 2008; $N_{2}=$ number of trees 2014; $P=$ pioneer; $C L=$ light-demanding climax; $C S=$ shade-tolerant climax.

Tabela 2 - Número de indivíduos, porcentagem (valores entre parênteses) de indivíduos mortos e recrutados, e mudança líquida em número de indivíduos (ChN) por guilda de regeneração em um fragmento de Floresta Ombrófila Mista Alto-Montana sob perturbações antrópicas crônicas, sendo: N1 = número de indivíduos em 2008; N2 = número de individuos em 2014; $P=$ pioneira; $C L=$ climax exigente em luz; $C S=$ clímax tolerante a sombra.

\begin{tabular}{lccccc}
\hline & $\mathrm{P}$ & $\mathrm{LC}$ & $\mathrm{SC}$ & Chisq & gl \\
\hline $\mathrm{N}_{1}$ & 252 & 939 & 147 & & \\
Dead* & $50(19.9 \%)$ & $81(8.6 \%)$ & $25(17.0 \%)$ & 29.08 & 2 \\
Recruited* & $37(15.6 \%)$ & $82(8.7 \%)$ & $11(8.3 \%)$ & 10.35 & $2.85 \mathrm{e}^{-07^{*}}$ \\
$\mathrm{~N}_{2}$ & 238 & 940 & 133 & $0.0057^{*}$ \\
$\mathrm{Ch}_{\mathrm{N}}$ & -5.88 & 0.11 & -10.53 & \\
\hline
\end{tabular}

chisq, $g l$ and $p$-value - Proportion test respectively indicating chi-square value, degree of freedom and significance.

Revista Árvore. 2017;41(3):e410312 
and the increase of late species representativeness (Oliveira Filho et al., 2007). Thus, the tendency to structural stability we have found indicates that the forest has not reached its structural climax, and, as mentioned before, there was a richness reduction, mainly in pioneer and rare species $(<2$ sampled individuals), and a decrease in the representativeness of shade-tolerant climax species (SC). In addition, it should be noted that species with the highest basal area gain mostly belong to the light-demanding climax group, which representativeness in the community has remained practically stable therefore suggesting that disturbing conditions are favoring this regeneration guild.

Results obtained here thus reinforce the idea that chronic anthropic disturbances potentially influence both species richness - as once noted by Lôbo et al. (2011) -, which favors the process of biological homogenization, and the successional trajectory of forests, by either limiting or slowing down the process of ecological succession (Clark and Covey, 2012; Zamorano-Elgueta et al., 2014; ArroyoRodríguez et al., 2015). In long term, a floristically more homogeneous forest is thus expected, consisting mainly of functional groups (light-demanding climax) and species (e.g. Myrceugenia glaucescens, Xylosma ciliatifolia, Drimys angustifolia and Araucaria angustifolia) somehow favored in this context of disturbances, which is mostly characterized by the presence of cattle. It is interesting to notice that Araucaria angustifolia, a light-demanding climax species in extinction and therefore immune to logging, has increased its number of individuals and basal area, which indicates a tolerance to these types of disturbances. On the other hand, the existing conditions do not seem to be favorable to shade-tolerant species, such as Dicksonia sellowiana.

The fact that there was no structural degradation process, which is characterized by a reduction in the number of trees and basal area, as once observed in other areas with chronic disturbances similar to those occurring in the forest under study (Machado and Oliveira Filho, 2010; Silva et al., 2011), suggests that disturbances intensity may be a determining factor and/or that forest formations may have different levels of resilience and resistance to disturbances. Considering the studies published by Orihuela et al. (2015) and Reis et al. (2014), one can indeed inferred that MOF is mainly formed by a group of stress- tolerant species with Araucaria angustifolia as one of its representatives. While Orihuela et al. (2015) highlight MOF's history of expansion over open areas so as to favor the occurrence of light-demanding and disturbancetolerant species, Reis et al. (2014) emphasize the influence of indigenous cultures which activities cause chronic disturbances so as to also favor species adapted to disturbances. In the same way as Mello and Peroni (2015) highlight the conservation value of the Caívas (ecotypes from a cultural landscape in the north of the State of Santa Catarina, Brazil), our results thus indicate that the traditional use of forest remnants can also be considered as an alternative form of soil use. Although the traditional use may impact the forest ecosystem through a process of biological homogenization, it is understood that the resulting areas may perform more ecological functions than areas exclusively used for agriculture, livestock or forestry purposes.

\section{CONCLUSION}

The hypothesis which forest remnants would suffer from a process of structural degradation followed by richness reduction has been partially accepted. Indeed, there were floristic impoverishment and reduced representativeness of late species, but the structural dynamics has indicated a tendency for stability. This pattern suggests that chronic disturbances actually have the potential to reduce richness therefore favoring a long-term biological homogenization process. However, since there was no structural degradation, we have concluded that the forest's resilience/resistance to chronic disturbances capacity is a determining factor in responding to such disturbances. From an economic, social and environmental point of view of the use of the landscape, it is understood that MOF forest remnants traditional use can be a maintenance mechanism for floristically impoverished forest areas which ecological value is higher than areas where soil use is exclusively intended for agrosilvopastoral practices. On the other hand, this context makes necessary to conserve fully protected areas in order to maintain non disturbance-tolerant species, such as shadetolerant climax species.

\section{ACKNOWLEDGEMENTS}

We thank to CNPq for funding a research grant on scientific productivity for both the eighth and ninth authors of this paper and to the land's owner for allowing its study. 


\section{REFERENCES}

Arroyo Rodríguez V, Melo FPL, Martínez-Ramos M, Bongers F, Chazdon RL, Meave JA. et al. Multiple successional pathways in human modified tropical landscapes: new insights from forest succession, forest fragmentation and landscape ecology research. Biological Reviews. [on line] 2015.

Baptista SR, Rudel TK. A re-emerging Atlantic forest? Urbanization, industrialization and the forest transition in Santa Catarina, southern Brazil. Environmental Conservation. 2006;33:195-202

Clark JA, Covey KR. Tree species richness and the logging of natural forests: A meta-analysis. Forest Ecology and Management. 2012;276:146-53.

Corlet RT. Tropical forest ecology in the Anthropocene. In: Köhl M, Pancel L editors. Tropical forestry handbook. Nova York: Springer; 2014. p.1-6.

Figueiredo Filho A, Dias AN, Stepka TF, Sawczuk AR. Crescimento, mortalidade, ingresso e distribuição diamétrica em Floresta Ombrófila Mista. Floresta. 2010;40:763-76.

Formento S, Schorn LA, Ramos RAB. Dinâmica estrutural arbórea de uma floresta ombrófila mista em Campo Belo do Sul, SC. Cerne. 2004;10:196-212.

Higuchi P, Silva AC, Almeida JA, Bortoluzzi RLC, Mantovani A, Ferreira TS. et al. Florística e estrutura do componente arbóreo e análise ambiental de um fragmento de Floresta Ombrófila Mista Alto-Montana no município de Painel, SC. Ciência Florestal. 2013;23:153-64.

Fundação Instituto Brasileiro de Geografia e Estatística - IBGE. Manual técnico da vegetação brasileira. Rio de Janeiro: 2012. 323p.

Korning J, Balslev H. Growth and mortality of trees in Amazonian tropical rain forest in Ecuador. Journal of Vegetation Science. 1994;5:77-86.

Kozera C, Dittrich VAO, Silva SM. Fitossociologia do componente arbóreo de um fragmento de Floresta Ombrófila Mista Montana, Curitiba, PR, BR. Floresta. 2006;36:225-37.
Lewis SL, Edwards DP, Galbraith D. Increasing human dominance of tropical forests. Science. 2015;349(6250):827-32.

Lôbo D, Leão T, Melo FPL, Santos AMM, Tabarelli M. Forest fragmentation drives Atlantic forest of northeastern Brazil to biotic homogenization. Diversity and Distributions. 2011;17:287-96.

Lugo AE. Forestry in the Anthropocene. Science. 2015;349(6250):771.

Machado ELM, Oliveira Filho AT. Spatial patterns of tree community dynamics are detectable in a small (4 ha) and disturbed fragment of the Brazilian Atlantic forest. Acta Botânica Brasilica. 2010;24:250-61.

Mello AJM, Peroni N. Cultural landscapes of the Araucaria Forest in northern plateau of Santa Catarina, Brazil. Journal of Ethnobiology and Ethnomedicine. 2015;11(51):1-14.

Mews HA, Marimon BS, Pinto JRR, Silvério DV. Dinâmica estrutural da comunidade lenhosa em floresta estacional semidecidual na transição cerrado-floresta amazônica, Mato Grosso, Brasil. Acta Botanica Brasilica. 201 1;25:845-57.

Oliveira Filho AT, Vilela EA, Carvalho DA, Gavilanes ML. Effects of soils and topography on the distribution of tree species in a tropical riverine forest in south-eastern Brazil. Journal of Tropical Ecology. 1994;10:483-508.

Oliveira Filho AT, Carvalho WAC, Machado ELM, Higuchi P, Appolinário V, Castro GC. et al. Dinâmica da comunidade e populações arbóreas da borda e interior de um remanescente florestal na Serra da Mantiqueira, Minas Gerais, em um intervalo de cinco anos (1999-2004). Revista Brasileira de Botânica. 2007;30:149-61.

Orihuela RLL, Peres CA, Mendes G, Jarenkow JA, Tabarelli M. Markedly divergent tree assemblage responses to tropical forest loss and fragmentation across a strong seasonality gradient. PloS one. 2015;10:e0136018.

R Core Team. R: A Language and Environment for Statistical Computing. Vienna: [acessed on: 24 set. 2015]. Available at: http://www.R-project.org

Revista Árvore. 2017;41(3):e410312 
Rech CCC, Silva AC, Higuchi P, Schimalski MB, Pscheidt F, Schmidt AB. et al. Avaliação da restauração florestal de uma APP degradada em Santa Catarina. Floresta eAmbiente. 2015;22:194-203.

Reis MS, Ladio A, Peroni N. Landscapes with Araucaria in South America: evidence for a cultural dimension. Ecology and Society. 2014;19:43.

Ribeiro MC, Metzger JP, Martensen AC, Ponzoni FJ, Hirota MM. The Brazilian Atlantic Forest: How much is left, and how is the remaining forest distributed? Implications for conservation. Biological Conservation. 2009;142:1141-53.

Salami B, Higuchi P, Silva AC, Ferreira TS, Marcon AK, Buzzi Junior F. et al. Influência de variáveis ambientais na dinâmica do componente arbóreo em um fragmento de Floresta Ombrófila Mista em Lages, SC. Scientia Forestalis. 2014;42:197-207.

Sampaio MB, Guarino ESG. Efeitos do pastoreio de bovinos na estrutura populacional de plantas em fragmentos de floresta ombrófila mista. Revista Árvore. 2007;31:1035-46.

Sanquetta CR, Pizatto W, Péllico Netto S, Figueiredo Filho A, Eisfeld RL. Dinâmica da composição florística de um fragmento de Floresta Ombrófila Mista no Centro-Sul do Paraná. Revista Ciências Exatas e Naturais. 2010;1:77-88.

Schorn LA, Galvão F. Dinâmica do estrato arbóreo em três estádios sucessionais de uma Floresta Ombrófila Densa em Blumenau, SC. Cerne. 2009;15:221-35.
Sheil D, Burslem DFRP, Alder D. The interpretation and misinterpretation of mortality rate measures. Journal of Ecology. 1995;83:331-3.

Sheil D, Jennings S, Savill P. Long-term permanent plot observations of vegetation dynamics in Budongo, a Ugandan rain forest. Journal of Tropical Ecology. 2000;16:865-82.

Silva AC, van den Berg D, Higuchi P, Nunes MH. Dinâmica de uma comunidade arbórea após enchente em fragmentos florestais no sul de Minas Gerais. Revista Árvore. 2011;35:883-93.

Silva AC, Higuchi P, Aguiar MD, Negrini M, Fert Neto J, Hess AF. Relações florísticas e fitossociologia de uma Floresta Ombrófila Mista Montana Secundária em Lages, Santa Catarina. Ciência Florestal. 2012;22:193-206.

Silva AC, Higuchi P, Negrini M, Grudtner A, Zech DF. Caracterização fitossociológica e fitogeográfica de um trecho de floresta ciliar em Alfredo Wagner, SC, como subsídio para restauração ecológica. Ciência Florestal. 2013;23:579-93.

Swaine MD, Whitmore TC. On the definition of ecological species groups in tropical rain forests. Plant Ecology. 1988;75:81-6.

Zamorano-Elgueta C, Cayuela L, Rey-Benayas JM, Donoso PJ, Geneletti D, Hobbs RJ. The differential influences of human-induced disturbances on tree regeneration community: a landscape approach. Ecosphere. 2014;5(7):art90. 